\title{
Anti-modified citrullinated vimentin antibody: a novel biomarker associated with cardiac systolic dysfunction in patients with rheumatoid arthritis
}

\author{
Somayye Norouzi ${ }^{1}$, Ali Javinani ${ }^{2}$, Arya Aminorroaya ${ }^{3}$ and Maryam Masoumi $i^{4^{*}}$ (i)
}

\begin{abstract}
Background: Studies have demonstrated that seropositive patients with rheumatoid arthritis (RA) are susceptible to cardiovascular diseases (CVDs). In this study, we aimed to determine the association of autoantibodies with the echocardiographic parameters of systolic and diastolic dysfunction in such patients.

Methods: In this cross-sectional study, we evaluated patients with RA who were referred to our clinic from October 2017 to August 2018. After the exclusion of patients with concomitant CVD, all patients underwent transthoracic echocardiography and measurement of plasma autoantibodies. Moreover, possible confounders-including medications, CVD risk factors, Framingham risk score, disease activity score-28, duration of disease, simple disease activity index, and functional status - were assessed.

Results: We studied 135 patients with RA (mean age $=52.3$ years; 111 (82.2\%) females). We had missing data rates of up to $8.9 \%$ for some characteristics. E velocity was inversely correlated with rheumatoid factor $(P=0.009)$. Furthermore, the plasma levels of anti-citrullinated protein and anti-modified citrullinated vimentin (anti-MCV) antibodies were negatively correlated with left ventricular ejection fraction (LVEF) $(P=0.019$ and $P<0.001$, respectively). After an adjustment for possible confounders, the linear regression model demonstrated that the antiMCV level and the patient's age are significant predictors of LVEF. The receiver operating characteristic curve showed that anti-MCV antibody titer $\geq 547.5(\mathrm{IU} / \mathrm{mL})$ signifies reduced LVEF $(<50 \%)$ with a sensitivity of $85.7 \%$ and specificity of $93 \%$ (C-statistic $=0.843$ ).

Conclusions: Our findings showed a significant inverse correlation between anti-MCV antibody titer and LVEF. These results indicate that the application of anti-MCV is promising for the screening and early detection of cardiac systolic dysfunction. Future prospective studies will determine its role.
\end{abstract}

Keywords: Autoantibodies, Anti-citrullinated protein antibodies, Anti-modified citrullinated vimentin, Anti-MCV, Arthritis, rheumatoid, Rheumatoid factor, Ejection fraction, Echocardiography

\footnotetext{
* Correspondence: mmasoomi@muq.ac.ir

${ }^{4}$ Clinical Research Development Center, Shahid Beheshti Hospital, Qom

University of Medical Sciences, Azadegan Sq., Shahid Beheshti Blvd, Qom,

Iran

Full list of author information is available at the end of the article
}

(c) The Author(s). 2020 Open Access This article is licensed under a Creative Commons Attribution 4.0 International License, which permits use, sharing, adaptation, distribution and reproduction in any medium or format, as long as you give appropriate credit to the original author(s) and the source, provide a link to the Creative Commons licence, and indicate if changes were made. The images or other third party material in this article are included in the article's Creative Commons licence, unless indicated otherwise in a credit line to the material. If material is not included in the article's Creative Commons licence and your intended use is not permitted by statutory regulation or exceeds the permitted use, you will need to obtain permission directly from the copyright holder. To view a copy of this licence, visit http://creativecommons.org/licenses/by/4.0/ The Creative Commons Public Domain Dedication waiver (http://creativecommons.org/publicdomain/zero/1.0/) applies to the data made available in this article, unless otherwise stated in a credit line to the data. 


\section{Background}

Rheumatoid arthritis (RA) is an auto-immune disorder that is mainly characterized by chronic synovitis and the progressive destruction of the joints. RA can involve nearly every organ in the body at a low prevalence when compared to its articular manifestations. According to the high prevalence and chronicity of RA, it has a significant socioeconomic burden [1]. The global disability-adjusted life year of RA has increased over the past decades, with approximately 3.5 million years in 2017, 0.14 of the global share [2]. Introducing novel diagnostic biomarkers, imaging studies, and effective medications have diminished the debilities of the disorder in the face of its increased prevalence [1].

The all-cause mortality rate of RA patients is significantly higher than that of the healthy population [1]. Most recent population-based studies have demonstrated that cardiovascular diseases (CVDs) are the leading cause of death among patients with RA [3, 4]. This can be attributed to the higher prevalence of conventional CVD risk factors among this population. In addition, certain RA-specific risk factors, including systemic inflammatory responses and cytokines, can worsen endothelial cell dysfunction and favor the formation of atherosclerotic plaques [5]. Moreover, the posttranscriptional modification of proteins, including citrullination, exposes some neo-antigens to the immune system. Anti-citrullinated protein antibodies (ACPAs) can react with these neo-antigens and activate an inflammatory cascade, thereby causing tissue damage [6].

According to the high morbidity and mortality of CVD among patients with RA, the European League Against Rheumatism (EULAR) recommended regular assessments of patients in this regard [7]. According to this guideline, symptoms such as prolonged disease duration, extra-articular manifestations, and positive ACPA and rheumatoid factor (RF) were considered as risk factors of CVD. Risk assessment for CVD can be done by certain clinical, laboratory, and imaging studies. Transthoracic echocardiography (TTE) is a reliable, non-invasive, and inexpensive method that can present signs of previous ischemia, myocardial systolic dysfunction, myocardial diastolic dysfunction, and valvular and pericardial abnormalities [8]. Owing to the high prevalence of echocardiographic markers of diastolic dysfunction among patients with RA, TTE seems to be valuable for the early detection of occult CVD [9].

As mentioned previously, seropositive RA patients are susceptible to CVDs. Moreover, RA-specific autoantibodies, especially ACPA, can trigger an inflammatory cascade against the citrullinated neo-antigens. Because patients with RA interstitium of the myocardium have high levels of citrullinated proteins compared to healthy individuals [10], these targeted inflammatory cascades could lead to ventricular systolic and diastolic dysfunction. In the present study, we have aimed to shed light on the correlation between RA-specific autoantibodies and TTE parameters. Moreover, we have investigated the correlation between anti-modified citrullinated vimentin (anti$\mathrm{MCV}$ ) antibody titer with ventricular dysfunction parameters, which has not been reported previously.

\section{Methods \\ Study population}

We conducted this cross-sectional study on patients with RA from our outpatient rheumatology clinic of Shahid Beheshti hospital, Qom University of Medical Sciences. Patients were selected by a simple random sampling approach, and all participants fulfilled the American college of rheumatology (ACR)/EULAR 2010 RA classification criteria [11]. Patients with a known history of overt CVDs, including coronary artery disease, peripheral arterial disease, cerebrovascular disease, dysrhythmia, and heart failure, were excluded from the study. Moreover, patients receiving biological treatment and cardiotoxic agents (cyclophosphamide, anthracyclines, and illicit drugs) were excluded. In cases of an indication of the primary prevention of CVDs with statins, patients were prescribed these medications. Furthermore, patients with hypertension were treated with anti-hypertensive medications. It should be noted that the participants did not receive any other cardiovascular medications like aspirin, nitrates, or betablockers. The study was performed from October 2017 to August 2018.

\section{Study design}

This paper investigated the connection between RAspecific autoantibodies and TTE variables regarding systolic and diastolic dysfunction. In order for the scientific results to be more accurate, we have considered certain confounding factors in our analyses that could independently influence the ventricular function and subsequently alter the TTE findings. The Framingham risk score was used to represent the traditional risk factors for CVD, including sex, age, smoking status, diabetes mellitus history, hypertension, and dyslipidemia [12]. The Framingham risk score was calculated using an online calculator (https://reference.medscape.com/calculator/framingham-cardiovascular-disease-risk). Patients were considered as having dyslipidemia if they met one of the following criteria: 1) use of lipid-lowering agents; 2) total cholesterol $\geq 200 \mathrm{mg} / \mathrm{dL}$; 3) low-density lipoprotein cholesterol $\geq 100 \mathrm{mg} / \mathrm{dL}$; 4) high-density lipoprotein $\leq 40 \mathrm{mg} / \mathrm{dL}$ for men or $50 \mathrm{mg} / \mathrm{dL}$ for women; 5) triglyceride $\geq 150 \mathrm{mg} / \mathrm{dL}$. Hypertension was defined as systolic blood pressure $\geq 140 \mathrm{mmHg}$ or diastolic blood pressure $\geq 90 \mathrm{mmHg}$. Moreover, the chronic inflammatory state of RA has a significant effect on a patient's cardiovascular risk profile. Not only the disease activity at the time of the study is a major contributor, but also 
the previous sequels of the disorder have noticeable effects on the cardiac system. Therefore, we have calculated the disease activity at the time of the study by considering the disease activity score-28/erythrocyte sedimentation rate (DAS-28/ESR) and a simple disease activity index (SDAI) for RA. According to these indices, all patients were in the remission phase during the study period. The ACR functional class of RA was calculated for considering sequels of the disease. The ACR functional classes demonstrate that the disability of patients occurred over years of suffering from RA. Among our patients with prolonged RA (disease duration $\geq 10$ years), $38.5 \%$ had an ACR functional class of I, which means that they were able to perform everyday activities without any limitations.

\section{Laboratory data}

Blood sampling was done at the time of the study. Similar laboratory kits were used to accurately assess the association of autoantibodies' quantitative titer with TTE variables. RF was measured by the agglutination method with a ENISON RF kit. ACPA and anti-MCV antibodies were measured with a CHORUS instrument via an enzymelinked immunoabsorbent assay and using CHORUS ACPA kit and ORGENTEC anti-MCV kit, respectively. The upper limits of ACPA and anti-MCV antibody measurements were $300 \mathrm{IU} / \mathrm{mL}$ and $1000 \mathrm{IU} / \mathrm{mL}$, respectively. ACPA titer up to $5 \mathrm{IU} / \mathrm{mL}$ was considered normal, and anti-MCV antibody titer $\geq 20 \mathrm{IU} / \mathrm{mL}$ was regarded as positive. In our study population, the RF, ACPA, and antiMCV were positive among $40.0 \%, 57.8 \%$, and $46.7 \%$ of the patients, respectively. ESR was also measured using the Westergren method and an IMPROVE autoanalyzer. Lipid profiles and fasting blood glucose levels were obtained from the patients' documents. These had been measured maximally 1 year prior to the study period. All the investigations were conducted in the hospital laboratory without any missing data.

\section{TTE}

All participants underwent TTE and tissue Doppler imaging, which was performed by an expert cardiologist (SN). The TTE was done by a commercially available instrument and a $2.5 \mathrm{MHz}$ probe. The study was performed on the left lateral decubitus position. We evaluated parameters related to cardiac systolic and diastolic dysfunction, including the left ventricular ejection fraction (LVEF), pulmonary arterial pressure (PAP), E velocity, E/e' ratio, tricuspid annular plane systolic excursion (TAPSE), isovolumic relaxation time (IVRT), and right ventricular systolic motion (RVSm). The LVEF was primarily determined visually, and abnormal values were rechecked via the Simpson method. Among our RA patients, 7 cases (5.2\%) had systolic dysfunction with an LVEF of less than $50 \%$.

\section{Statistical analysis}

Data analysis was conducted using SPSS software (IBM Corp. Released 2010. IBM SPSS Statistics for Windows, Version 19.0. Armonk, NY: IBM Corp.). Continuous variables were reported as means \pm standard deviation (SD). Medians with interquartile ranges (IQRs) were used to describe nonnormally distributed variables. Finally, categorical variables were presented as numbers and percentages. We used Pearson's correlation coefficient to evaluate the associations between two continuous variables. Correlations between ordinal and continuous variables were measured based on Spearman's rho correlation coefficient. A linear regression model was applied to control and adjust the effects of possible confounders and prevent the results from becoming biased.

Furthermore, we determined the power of anti-MCV titer in the prediction of reduced LVEF (LVEF $<50 \%)$ using the receiver operating characteristic (ROC) curve analysis. This was done by plotting sensitivity against (1 - specificity) and calculating the area under the curve (C-statistic) as the prediction power. We also calculated a cut-off value for anti-MCV to discriminate between reduced $(\mathrm{LVEF}<50 \%)$ and preserved LVEF (LVEF $\geq 50 \%$ ). The value that was correlated with the highest sensitivity and specificity was used as the cut-off value.

\section{Results}

We studied 135 patients with RA (mean age $=52.3$ years; 111 (82.2\%) females) (Table 1). Their baseline characteristics and echocardiographic parameters are presented in Table 1. Most of the data were complete; however, IVRT is missing for $12(8.9 \%)$ cases (Table 1$)$. All included patients received 2.5-7.5 $\mathrm{mg}$ of prednisolone per day.

Among all evaluated echocardiographic variables, E velocity showed a statistically significant inverse correlation with RF (Spearman's rho coefficient $=-0.223$, $P=0.009$ ). Furthermore, ACPA and anti-MCV demonstrated similar associations with LVEF (Pearson's coefficient $=-0.201, P=0.019$; Pearson's coefficient $=$ - 0.322, $P<0.001$, respectively). We did not find any other statistically significant correlations between RF, ACPA, or anti-MCV with assessed echocardiographic indices (Table 2).

To consider possible confounding effects, a simple linear regression model was fitted to the data to predict LVEF based on anti-MCV, ACPA, and all possible confounders, including the Framingham score. A significant regression model was constructed $(\mathrm{F}(2,128)=12.429$, $P<0.001)$ with an $\mathrm{R}$-square of 0.163 . In this model, antiMCV and age were significant predictors of LVEF; nonetheless, the association between LVEF and ACPA did not remain significant after the adjustment for possible confounders (Table 3).

A ROC curve was plotted to predict reduced LVEF $(\mathrm{LVEF}<50 \%)$ based on the anti-MCV titer. The area 
Table 1 Baseline characteristics and echocardiographic parameters of participants

\begin{tabular}{|c|c|c|}
\hline Characteristic & Value $^{a}$ & Missing data ${ }^{a}$ \\
\hline \multicolumn{3}{|l|}{ Demographics } \\
\hline Age (year) & $52.3 \pm 11.9$ & $0(0 \%)$ \\
\hline Female sex & $111(82.2 \%)$ & $0(0 \%)$ \\
\hline Female to male ratio & 4.6 & $0(0 \%)$ \\
\hline Height (meter) & $1.58 \pm 0.08$ & $0(0 \%)$ \\
\hline Weight (kilogram) & $71.0 \pm 13.8$ & $3(2.2 \%)$ \\
\hline Body mass index $\left(\mathrm{kg} / \mathrm{m}^{2}\right)$ & $28.6 \pm 5.3$ & $3(2.2 \%)$ \\
\hline Duration of disease (year) & $9.7 \pm 9.4$ & $0(0 \%)$ \\
\hline DAS-28/ESR & $2.1 \pm 0.4$ & $1(0.7 \%)$ \\
\hline SDAl & $2.7 \pm 0.7$ & $1(0.7 \%)$ \\
\hline ACR functional class & $1[1]$ & $1(0.7 \%)$ \\
\hline Dyslipidemia & $30(22.2 \%)$ & $0(0 \%)$ \\
\hline Diabetes mellitus & $27(20.0 \%)$ & $0(0 \%)$ \\
\hline Hypertension & $35(25.9 \%)$ & $0(0 \%)$ \\
\hline Framingham score (\%) & $2.7 \pm 4.1$ & $0(0 \%)$ \\
\hline \multicolumn{3}{|l|}{ Medications } \\
\hline Prednisolone & $135(100 \%)$ & $0(0 \%)$ \\
\hline Hydroxychloroquine & $128(94.8 \%)$ & $0(0 \%)$ \\
\hline Methotrexate & $125(92.6 \%)$ & $0(0 \%)$ \\
\hline Leflunomide & $36(26.7 \%)$ & $0(0 \%)$ \\
\hline Sulfasalazine & $30(22.2 \%)$ & $0(0 \%)$ \\
\hline \multicolumn{3}{|l|}{ Laboratory data } \\
\hline $\mathrm{RF}(\mathrm{IU} / \mathrm{mL})$ & $0[2]$ & $0(0 \%)$ \\
\hline ACPA (IU/mL) & $91.5 \pm 115.9$ & $0(0 \%)$ \\
\hline Anti-MCV (IU/mL) & $150.1 \pm 290.0$ & $0(0 \%)$ \\
\hline Total cholesterol (mg/dL) & $182.6 \pm 33.9$ & $0(0 \%)$ \\
\hline Low-density lipoprotein cholesterol (mg/dL) & $106.9 \pm 31.1$ & $0(0 \%)$ \\
\hline High-density lipoprotein cholesterol (mg/dL) & $50.3 \pm 11.9$ & $0(0 \%)$ \\
\hline Triglyceride (mg/dL) & $137.6 \pm 57.4$ & $0(0 \%)$ \\
\hline \multicolumn{3}{|l|}{ Echocardiographic indices } \\
\hline LVEF (\%) & $57.0 \pm 5.4$ & $0(0 \%)$ \\
\hline PAP $(\mathrm{mmHg})$ & $26.7 \pm 7.8$ & $10(7.4 \%)$ \\
\hline E velocity (m/second) & $0.8 \pm 0.2$ & $0(0 \%)$ \\
\hline E/e' ratio & $9.8 \pm 4.9$ & $3(2.2 \%)$ \\
\hline TAPSE (mm) & $23.2 \pm 3.1$ & $5(3.7 \%)$ \\
\hline IVRT (millisecond) & $125.4 \pm 19.1$ & $12(8.9 \%)$ \\
\hline $\operatorname{RVSm}(\mathrm{cm} / \mathrm{s})$ & $12.3 \pm 1.8$ & $10(7.4 \%)$ \\
\hline
\end{tabular}

ACPA Anti-citrullinated protein antibody, ACR American college of rheumatology, Anti-MCV Anti-modified citrullinated vimentin, DAS-28/ESR Disease activity score28/erythrocyte sedimentation rate, IVRT Isovolumic relaxation time, LVEF Left ventricular ejection fraction, PAP Pulmonary arterial pressure, RF Rheumatoid factor RVSm Right ventricular systolic motion, SDAI Simple disease activity index, TAPSE Tricuspid annular plane systolic excursion

${ }^{a}$ Data are reported as Mean \pm SD, Median $[I Q R]$, or Number (Proportion\%)

under the curve, which represents the strength of the prediction, was 0.843 (95\% confidence interval: $0.631_{-}$ $1.000, P=0.002)$. An anti-MCV titer of greater than or equal to $547.5 \mathrm{IU} / \mathrm{mL}$ identified reduced LVEF with a sensitivity of $85.7 \%$ and a specificity of $93.0 \%$.

\section{Discussion}

We have investigated the correlation between autoantibodies and echocardiographic findings in patients with RA who were asymptomatic for CVDs. To more accurately estimate the influence of autoantibodies per se on 
Table 2 Association of echocardiographic parameters with auto-antibodies

\begin{tabular}{|c|c|c|c|c|}
\hline \multicolumn{2}{|l|}{ Parameter } & \multirow{2}{*}{$\frac{R F}{-0.161^{a}}$} & \multirow{2}{*}{$\frac{\text { ACPA }}{-0.201^{b}}$} & \multirow{2}{*}{$\frac{\text { Anti-MCV }}{-0.322^{b}}$} \\
\hline LVEF (\%) & Coefficient & & & \\
\hline & $P$ & 0.061 & 0.019 & $<0.001$ \\
\hline \multirow[t]{2}{*}{ PAP (mmHg) } & Coefficient & $0.060^{a}$ & $-0.036^{b}$ & $0.077^{b}$ \\
\hline & $P$ & 0.504 & 0.692 & 0.393 \\
\hline \multirow[t]{2}{*}{ E velocity (m/second) } & Coefficient & $-0.223^{a}$ & $-0.011^{b}$ & $0.083^{b}$ \\
\hline & $P$ & 0.009 & 0.903 & 0.336 \\
\hline \multirow[t]{2}{*}{ E/e' ratio } & Coefficient & $0.128^{a}$ & $0.045^{b}$ & $0.097^{b}$ \\
\hline & $P$ & 0.144 & 0.610 & 0.268 \\
\hline \multirow[t]{3}{*}{ TAPSE (mm) } & Coefficient & $-0.142^{a}$ & $-0.092^{b}$ & $0.078^{b}$ \\
\hline & $P$ & 0.106 & 0.296 & 0.376 \\
\hline & $P$ & 0.311 & 0.546 & 0.716 \\
\hline \multirow[t]{2}{*}{ IVRT (millisecond) } & Coefficient & $0.028^{a}$ & $0.058^{b}$ & $0.128^{b}$ \\
\hline & $P$ & 0.759 & 0.523 & 0.157 \\
\hline \multirow[t]{2}{*}{ RVSm } & Coefficient & $0.008^{a}$ & $-0.021^{b}$ & $0.012^{b}$ \\
\hline & $P$ & 0.927 & 0.817 & 0.897 \\
\hline
\end{tabular}

ACPA Anti-citrullinated protein antibody, Anti-MCV Anti-modified citrullinated vimentin, IVRT Isovolumic relaxation time, LVEF Left ventricular ejection fraction, PAP Pulmonary arterial pressure, RF Rheumatoid factor, RVSm Right ventricular systolic motion, TAPSE Tricuspid annular plane systolic excursion aspearman's rho correlation coefficient

${ }^{b}$ Pearson correlation coefficient

cardiac imaging findings, the confounding effects of demographic features, medications, atherosclerosis, and disease duration were eliminated by multivariate regression analysis. In addition, the cumulative undesirable effects of a prolonged inflammatory state of RA were also considered in the analyses using the ACR functional class of RA. Our findings showed that the anti-MCV antibody and RF titers were associated with reduced LVEF and E velocity, respectively; however, we did not identify any significant correlation between echocardiographic findings and ACPA level.

Anti-MCV is an antibody that protects against citrullinated vimentin. Therefore, it is categorized as an ACPA. It is primarily identified as a diagnostic marker in RA, but its sensitivity seems not to be significantly different from non-specific ACPA [13]. However, some added value of its concomitant measurement with ACPA was proposed to augment the sensitivity of early RA diagnosis [14]. Nevertheless, limited studies have gone beyond

Table 3 Linear regression analysis for prediction of LVEF

\begin{tabular}{lll}
\hline Variable & Multivariate analysis & \\
\cline { 2 - 3 } & Coefficient $(95 \%$ confidence interval) & $P$ \\
\hline Anti-MCV & $-0.006\left(-0.009 \_-0.003\right)$ & $<0.001$ \\
Age (year) & $-0.111\left(-0.186 \_-0.037\right)$ & 0.004 \\
Constant & $63.604\left(59.577 \_67.632\right)$ & $<0.001$ \\
\hline
\end{tabular}

Anti-MCV Anti-modified citrullinated vimentin the diagnostic value of anti-MCV antibody to investigate its clinical value in RA.

Notably, it was demonstrated that anti-MCV antibody level was associated with inflammatory markers (ESR and C-reactive protein), disease activity, and carotid intima-media thickness (CIMT) in treatment-naïve RA cases [15]. In addition, the anti-MCV antibody titer was diminished after treatment commencement, and its changes were correlated with changes in cardiovascular risk factors including CIMT, total cholesterol, lowdensity lipoprotein cholesterol/high-density lipoprotein cholesterol, interleukin 6 , and tumor necrosis factoralpha [15]. Nonetheless, no association was identified between ACPA changes and the markers mentioned above. This finding could indicate the pathogenic role of anti$\mathrm{MCV}$ antibodies in early precocious atherosclerosis among patients with RA [15].

The negative association between anti-MCV antibody titer and LVEF could be justified by a certain hypothesis. Giles and colleagues found a significantly higher level of citrullinated proteins in the interstitium of the myocardium of patients with RA than in healthy individuals [10]. Moreover, they demonstrated that myocardial fibrosis was associated with high citrullination scores in RA myocardium slides [10]. Surprisingly, the citrullination of sarcomeric proteins, including vimentins, was demonstrated in the myocardium of patients with heart failure [16], and this process can significantly diminish the activity and contraction of cardiac sarcomeres [6]. Taken together, these observations provide strong but still inconclusive evidence that antibodies against citrullinated cardiac proteins induce inflammatory and destructive damage to the myocardium and further modify the cardiac function in patients with RA. This hypothesis is prominently in line with the association between antiMCV titer and LVEF.

The results obtained in the present study did not reveal any correlations between ACPA level and echocardiographic variables. Similar to our findings, several studies have also reported that there is no significant correlation between ACPA and LV systolic failure as defined by reduced LVEF and LV geometrical changes toward concentric remodeling [17-19]. In addition, most previous studies did not support the association of ACPA with diastolic dysfunction and LV mass index [20-23]. However, Logstrup et al. found a correlation between LV systolic dysfunction and ACPA in drugnaive RA patients with normal LVEF after a two-year follow-up [24]. They assessed LV function by conventional echocardiography and speckle-tracking echocardiography and demonstrated that patients with persistently elevated ACPA over 2 years exhibited smaller improvements in S' and worsened global longitudinal systolic strain (GLS) [24]. Changes in GLS and 
ACPA titer were also significantly correlated during the follow-up period [24]. In summary, it appears that this discrepancy comes from the different antibodies measured by each ACPA laboratory kit. We hypothesize that certain ACPAs that target citrullinated proteins of the myocardium have cardiotoxic effects, whereas the details of antibody subgroups have not been identified in previously mentioned studies.

The present study was subject to a number of potential methodological weaknesses. Due to the crosssectional design of the study, we could not assess the causal relationship between the anti-MCV antibody titer and diminished cardiac systolic function. This issue warrants future prospective studies to address this shortcoming. Moreover, we had missing data (up to $8.9 \%$ ) for some echocardiographic indices. In addition, we did not assess some echocardiographic parameters that are necessary to determine the exact grading of diastolic dysfunction, like left atrial size and index. Thus, we could not reliably examine the association between autoantibodies and cardiac diastolic dysfunction. Moreover, we did not evaluate serum markers of heart failure, such as $\mathrm{N}$-terminal pro-B-type natriuretic peptide, which can be helpful. In addition, participants' long-term use of medications might have affected our findings. This potential problem could be addressed by calculating the cumulative dose. The paucity of data regarding this issue is considered as another limitation of this study. Future prospective controlled studies that address the shortcomings of the present work are strongly encouraged.

\section{Conclusions}

To the best of our knowledge, this is the first study that has attempted to determine the association between antiMCV antibody level and the systolic function of the heart in patients with RA. Although the inverse correlation of anti-MCV antibody titer with LVEF may have pathophysiologic justifications, future prospective studies will determine the role of this antibody in the screening and early detection of cardiac systolic dysfunction in RA patients.

\section{Supplementary information}

Supplementary information accompanies this paper at https://doi.org/10. 1186/s12872-020-01676-x.

Additional file 1. Analyzed Data.

\section{Abbreviations}

ACPA: Anti-citrullinated protein antibody; ACR: American college of rheumatology; Anti-MCV: Anti-modified citrullinated vimentin; CIMT: Carotid intima-media thickness; CVD: Cardiovascular disease; DAS-28/ESR: Disease activity score-28/erythrocyte sedimentation rate; EULAR: European league against rheumatism; GLS: Global longitudinal systolic strain; IQR: Interquartile range; IVRT: Isovolumic relaxation time; LVEF: Left ventricular ejection fraction; PAP: Pulmonary arterial pressure; RA: Rheumatoid arthritis; RF: Rheumatoid factor; ROC: Receiver operating characteristic; RVSm: Right ventricular systolic motion; SD: Standard deviation; SDAl: Simple disease activity index; TAPSE: Tricuspid annular plane systolic excursion; TTE: Transthoracic echocardiography

\section{Acknowledgments}

Not applicable.

\section{Authors' contributions}

$\mathrm{SN}$ and MM contributed to the conception and design of the study. SN, AJ, $A A$, and MM contributed to the acquisition of data. AJ and AA contributed to the analysis and interpretation of data. AJ and AA drafted the manuscript. SN and MM critically revised the manuscript. SN, AJ, AA, and MM read and approved the final manuscript.

\section{Funding}

None.

\section{Availability of data and materials}

The dataset supporting the conclusions of this article is included within the article and its additional file.

\section{Ethics approval and consent to participate}

All procedures performed in this study were in accordance with the 2013 Helsinki Declaration. The protocol of this study was submitted and approved by the ethics committee of Qom University of Medical Sciences with the ID of IR.MUQ.REC.1398.034. We obtained written informed consent from all of the participants prior to inclusion in the study.

Consent for publication

Not applicable.

\section{Competing interests}

The authors declare that they have no competing interests.

\section{Author details}

'Department of Internal Medicine, Qom University of Medical Sciences, Qom, Iran. ${ }^{2}$ Rheumatology Research Center, Tehran University of Medical Sciences, Tehran, Iran. ${ }^{3}$ Tehran Heart Center, Tehran University of Medical Sciences, Tehran, Iran. ${ }^{4}$ Clinical Research Development Center, Shahid Beheshti Hospital, Qom University of Medical Sciences, Azadegan Sq., Shahid Beheshti Blvd, Qom, Iran

Received: 2 March 2020 Accepted: 19 August 2020

Published online: 26 August 2020

\section{References}

1. Minichiello E, Semerano $L$, Boissier MC. Time trends in the incidence, prevalence, and severity of rheumatoid arthritis: a systematic literature review. Joint Bone Spine. 2016:83(6):625-30.

2. Cross M, Smith E, Hoy D, Carmona L, Wolfe F, Vos T, Williams B, Gabriel S, Lassere $\mathrm{M}$, Johns $\mathrm{N}$, et al. The global burden of rheumatoid arthritis: estimates from the global burden of disease 2010 study. Ann Rheum Dis. 2014;73(7):1316-22.

3. Widdifield J, Paterson JM, Huang A, Bernatsky S. Causes of death in rheumatoid arthritis: how do they compare to the general population? Arthritis Care Res. 2018:70(12):1748-55.

4. Ometto F, Fedeli U, Schievano E, Botsios C, Punzi L, Corti MC. Cause-specific mortality in a large population-based cohort of patients with rheumatoid arthritis in Italy. Clin Exp Rheumatol. 2018;36(4):636-42.

5. England BR, Thiele GM, Anderson DR, Mikuls TR. Increased cardiovascular risk in rheumatoid arthritis: mechanisms and implications. BMJ. 2018;361: k1036.

6. Fert-Bober J, Crowgey EL, Sokolove J, Giles JT, Bathon JM, Van Eyk JE. The significance of myofilament protein citrullination in heart failure: citrullination in cardiovascular diseases. In: Nicholas AP, Bhattacharya SK, Thompson PR, editors. Protein deimination in human health and disease. Cham: Springer International Publishing; 2017. p. 205-25.

7. Agca R, Heslinga SC, Rollefstad S, Heslinga M, Mclnnes IB, Peters MJL, Kvien TK, Dougados M, Radner $H$, Atzeni $F$, et al. EULAR recommendations for cardiovascular disease risk management in patients with rheumatoid 
arthritis and other forms of inflammatory joint disorders: 2015/2016 update. Ann Rheum Dis. 2017;76(1):17-28.

8. Atzeni F, Corda M, Gianturco L, Porcu M, Sarzi-Puttini P, Turiel M. Cardiovascular imaging techniques in systemic rheumatic diseases. Front Med. 2018:5:26.

9. Aslam F, Bandeali SJ, Khan NA, Alam M. Diastolic dysfunction in rheumatoid arthritis: a meta-analysis and systematic review. Arthritis Care Res. 2013;65(4):534-43.

10. Giles JT, Fert-Bober J, Park JK, Bingham CO, Andrade F, Fox-Talbot K, Pappas $\mathrm{D}$, Rosen A, van Eyk J, Bathon JM, et al. Myocardial citrullination in rheumatoid arthritis: a correlative histopathologic study. Arthritis Res Ther. 2012;14(1):R39

11. Kay J, Upchurch KS. ACR/EULAR 2010 rheumatoid arthritis classification criteria. Rheumatology. 2012;51(suppl_6):vi5-9.

12. D'Agostino RB Sr, Vasan RS, Pencina MJ, Wolf PA, Cobain M, Massaro JM, Kannel WB. General cardiovascular risk profile for use in primary care: the Framingham heart study. Circulation. 2008;117(6):743-53.

13. Maraina $\mathrm{CH}$, Nurdayana AK, Rusni D, Azwany Y. Diagnostic value of antimodified citrullinated vimentin in rheumatoid arthritis. Int J Rheum Dis. 2010;13(4):335-9.

14. Diaz-Toscano ML, Olivas-Flores EM, Zavaleta-Muniz SA, Gamez-Nava J, Cardona-Munoz EG, Ponce-Guarneros M, Castro-Contreras U, Nava A, Salazar-Paramo M, Celis A, et al. Comparison of two assays to determine anti-citrullinated peptide antibodies in rheumatoid arthritis in relation to other chronic inflammatory rheumatic diseases: assaying anti-modified citrullinated vimentin antibodies adds value to second-generation anticitrullinated cyclic peptides testing. BioMed Res Int. 2014;2014:198198.

15. El-Barbary AM, Kassem EM, El-Sergany MA, Essa SA, Eltomey MA. Association of anti-modified citrullinated vimentin with subclinical atherosclerosis in early rheumatoid arthritis compared with anti-cyclic citrullinated peptide. J Rheumatol. 2011;38(5):828-34.

16. Fert-Bober J, Giles JT, Holewinski RJ, Kirk JA, Uhrigshardt H, Crowgey EL, Andrade F, Bingham CO 3rd, Park JK, Halushka MK, et al. Citrullination of myofilament proteins in heart failure. Cardiovasc Res. 2015;108(2):232-42.

17. Cioffi G, Viapiana O, Ognibeni F, Dalbeni A, Gatti D, Mazzone C, Faganello G, Di Lenarda A, Adami S, Rossini M. Combined circumferential and longitudinal left ventricular systolic dysfunction in patients with rheumatoid arthritis without overt cardiac disease. J Am Soc Echocardiogr. 2016;29(7):689-98,

18. Vizzardi E, Cavazzana I, Bazzani C, Pezzali N, Ceribelli A, Bonadei I, Franceschini F, D'Aloia A, Metra M, Tincani A, et al. Echocardiographic evaluation of asymptomatic patients affected by rheumatoid arthritis. J Investig Med. 2012;60(8):1204-8.

19. Giles JT, Malayeri AA, Fernandes V, Post W, Blumenthal RS, Bluemke D, Vogel-Claussen J, Szklo M, Petri M, Gelber AC, et al. Left ventricular structure and function in patients with rheumatoid arthritis, as assessed by cardiac magnetic resonance imaging. Arthritis Rheum. 2010;62(4):940-51.

20. Midtbo H, Gerdts E, Kvien TK, Olsen IC, Hirth A, Davidsen ES, Semb AG. Disease activity and left ventricular structure in patients with rheumatoid arthritis. Rheumatology. 2015;54(3):511-9.

21. Sharma A, Kaushik R, Kaushik RM, Kakkar R. Echocardiographic evaluation of diastolic dysfunction in rheumatoid arthritis - a case-control study. Mod Rheumatol. 2015;25(4):552-7.

22. Rudominer RL, Roman MJ, Devereux RB, Paget SA, Schwartz JE, Lockshin MD, Crow MK, Sammaritano L, Levine DM, Salmon JE. Independent association of rheumatoid arthritis with increased left ventricular mass but not with reduced ejection fraction. Arthritis Rheum. 2009;60(1):22-9.

23. Davis JM 3rd, Lin G, Oh JK, Crowson CS, Achenbach SJ, Therneau TM, Matteson EL, Rodeheffer RJ, Gabriel SE. Five-year changes in cardiac structure and function in patients with rheumatoid arthritis compared with the general population. Int J Cardiol. 2017;240:379-85.

24. Logstrup BB, Masic D, Laurbjerg TB, Blegvad J, Herly M, Kristensen LD, Urbonaviciene G, Hedemann-Nielsen A, Ellingsen T. Left ventricular function at two-year follow-up in treatment-naive rheumatoid arthritis patients is associated with anti-cyclic citrullinated peptide antibody status: a cohort study. Scand J Rheumatol. 2017;46(6):432-40.

\section{Publisher's Note}

Springer Nature remains neutral with regard to jurisdictional claims in published maps and institutional affiliations.

Ready to submit your research? Choose BMC and benefit from:
- fast, convenient online submission
- thorough peer review by experienced researchers in your field
- rapid publication on acceptance
- support for research data, including large and complex data types
- gold Open Access which fosters wider collaboration and increased citations
- maximum visibility for your research: over 100M website views per year
At BMC, research is always in progress.
Learn more biomedcentral.com/submissions

